\title{
P-31 Nuclear Magnetic Resonance Spectroscopic Study of Obstructive Uropathy in the Rat
}

\author{
Joseph I. Shapiro and Laurence Chan \\ Department of Medicine, University of Colorado Health Sciences Center, Denver, Colorado 80262
}

\begin{abstract}
P-31 nuclear magnetic resonance (NMR) spectroscopy of the rat kidney with ureteral ligation resulted in a rapid and major increase in a peak resonating at $7096.63 \pm 0.65 \mathrm{~Hz}$ from the reference frequency of phosphorus $(32.60 \mathrm{MHz})$. This corresponded to an increase in the concentration of the substance responsible for peak $X$ from $0.34 \pm 0.04 \mu \mathrm{mol} / \mathrm{g}$ wet weight in normal kidneys to $1.45 \pm 0.27 \mu \mathrm{mol} / \mathrm{g}$ wet weight in unilaterally obstructed kidneys and $2.00 \pm 0.34 \mu \mathrm{mol} / \mathrm{g}$ wet weight in bilaterally obstructed kidneys at $3 \mathrm{~h}(P<0.01)$. Further NMR studies performed with in vivo kidneys and tissue extracts revealed that inorganic phosphate in the urine, resonating at a lower frequency due to the acid pH environment, was responsible for the increase in this peak. These findings may prove to be of fundamental interest as well as potential clinical significance.
\end{abstract}

\section{Introduction}

Phosphorus-31 nuclear magnetic resonance (NMR) ${ }^{1}$ has been used to study changes in the concentrations of high energy phosphate compounds and intracellular $\mathrm{pH}$ on isolated cells and tissues in the early 1970 s $(1,2)$. More recently, P-31 NMR spectroscopy has been successfully used to study renal biochemistry, a topic which has been extensively reviewed (3-5). Specifically, this technique has been used to study normal renal energy metabolism as well as cellular biochemical changes occurring with systemic hypotension and ischemia, and also to study changes in extracellular acid-base status in the kidney (6-12).

When a kidney is subjected to ureteral ligation, there are major changes in renal blood flow as well as net tubular sodium reabsorption (13-19). Because of the changes in substrate supply and energy demand, we reasoned that there might be associated changes in the concentrations of high energy phosphate compounds in the kidney that occur in ureteral ligation which could be studied with P-31 NMR.

In this paper, we report dramatic changes in the P-31 NMR spectra of rat kidneys subjected to ureteral ligation.

Address reprint requests to Dr. Chan, Division of Renal Diseases, University of Colorado Health Sciences Center, 4500 E. Ninth Ave., Box C281, Denver, CO 80262. 1987.

Received for publication 20 August 1986 and in revised form 8 July

1. Abbreviations used in this paper: NMR, nuclear magnetic resonance; $P i$, inorganic phosphate; $T_{1}$, spin-lattice relaxation time.

J. Clin. Invest.

(c) The American Society for Clinical Investigation, Inc. $0021-9738 / 87 / 11 / 1422 / 06 \quad \$ 2.00$

Volume 80, November 1987, 1422-1427

\section{Methods}

\section{Animals}

Male Sprague-Dawley rats (300-350 g) obtained from a commercial supplier (Sasco, Omaha, NE), fed on standard rat chow (Ralston-Purina Co., St. Louis, MO) and allowed free access to tap water, were used. Rats were anesthetized with pentobarbital administered intraperitoneally and then subjected to a flank incision (unilateral or bilateral). Additional anesthesia was given to the rats as needed during the experiment and postoperative period to relieve their distress. The kidney was exposed and mobilized using blunt dissection. Ureteral ligation was accomplished using two $4-0$ silk ties tied $\sim 1.0 \mathrm{~cm}$ from the renal hilus. Release of obstruction was accomplished by ureteral cannulation proximal to the point of obstruction using P-50 tubing. Urine obtained by this cannulation was studied for $\mathrm{pH}$ determination, phosphate concentration, and spin-lattice relaxation time $\left(T_{1}\right)$ determination (see below). The rat kidneys studied by NMR spectroscopy were externalized after the flank incision, separated from the muscle and skin with surgical gauze, and covered with a thin plastic covering. Care was taken during this procedure to avoid compromising the vascular supply of the kidney.

\section{P-31 NMR spectroscopy}

In vivo P-31 NMR spectra were obtained using a 1.89 Tesla $30-\mathrm{cm}$ horizontal bore cryomagnet (Oxford Research Systems) and a Biospec spectrometer (Bruker Instruments, Inc., Billerica, MA). The transmitter and receiver used was a $1.5-\mathrm{cm}$ diam 2-turn solenoid tuned to the resonance frequency of phosphate in this magnet $(32.60 \mathrm{MHz})$, which was built by the authors. The $90^{\circ}$ and $180^{\circ}$ pulse widths ( 15 and $30 \mu \mathrm{s}$, respectively) were determined on phantoms containing $100 \mathrm{mM}$ inorganic phosphate $(\mathrm{Pi})$ by testing the effect of increasing pulse widths on the resultant P-31 NMR spectra using one scan for each pulse width studied. The accuracy of these pulse widths were later confirmed using kidney tissue in situ. Kidney spectra were obtained by placing the solenoid over the externalized rat kidney. Spectra used for comparison were obtained using 512 scans each using a $60^{\circ}$ pulse and a 2-s delay. Spectroscopy of urine was performed on $2-\mathrm{ml}$ urine samples placed in small plastic tubes positioned within the same probe described above. Spectra of these urine samples were obtained with 32 scans of $60^{\circ}$ using 2-s delays. $T_{1}$ values for the spectral peaks of the in situ kidneys and the major spectral peak of urine were obtained using the inversion recovery method $(1,2,6)$.

P-31 NMR spectra from extracts (see below) were performed on a 4.7 Tesla vertical bore cryomagnet (Oxford Instruments, Oxford, England) using a 200-MHz spectrometer (Bruker Instruments, Inc.) and a commercial probe tuned to the resonance frequency of $\mathrm{Pi}$ in this magnet $(80.85 \mathrm{MHz})$. Spectra from extracts are plotted as parts per million $(\mathrm{ppm})$ from the resonance frequency of phosphoric acid.

\section{Destructive chemical analysis}

Kidneys from animals were freeze clamped using Wollenberger clamps cooled in liquid nitrogen and stored at $-70^{\circ} \mathrm{C}$ before extraction. Extractions were performed using $10 \%$ perchloric acid, which was followed by centrifugation for $25 \mathrm{~min}$ at $12,000 \mathrm{~g}$ and neutralization of the supernatant with $30 \%$ potassium hydroxide. The neutralized extract was assayed for ATP using an enzymatic method that has been well described (20). Urine phosphate was determined using a standard 
chemical assay (21). Urine $\mathrm{pH}$ was determined using a pH meter (Beckman Instruments Inc., Palo Alto).

\section{Quantitation}

P-31 NMR spectral peaks from spectra obtained from in vivo samples and extracts were quantitated by relative areas under the spectral peaks. Exponential multiplication was applied to the free induction decay with a line broadening of $10 \mathrm{~Hz}$. Because of the broad phospholipid signal present on the in vivo spectra, these spectra were baseline corrected by deconvolution before peak area calculations. For each experiment, the $B_{0}$ field was shimmed using the proton signal of the sample and was brought to resonance at $80.55 \mathrm{MHz}$. Phosphate resonances are expressed as $\mathrm{Hz}$ from $32.60 \mathrm{MHz}$. The chemical environment of phosphate associated with the various spectral peaks was for the most part as has been previously described $(1,2)$. The peak at $\sim 7,096 \mathrm{~Hz}$ is referred to as peak $\mathrm{X}$. The absolute molar concentration of the phosphate resonating at this frequency was calculated by multiplying the relative area of peak $X$ divided by the relative area of the beta ATP peak, by the absolute molar concentration of ATP determined by enzymatic analysis performed on the extract of that kidney (22).

\section{Experimental design}

Time course. Initially, serial spectroscopy on several animals treated with either unilateral or bilateral ureteral ligation was performed recording spectra every $20 \mathrm{~min}$ for the first $16-24 \mathrm{~h}$ after ureteral ligation. Using the data gathered from these studies, it was learned that maximal spectral changes occurred from 3 to $4 \mathrm{~h}$ after ligation. Therefore rats were assigned to the following groups. A, no obstruction; B, unilateral obstruction for $3 \mathrm{~h}$; C, bilateral obstruction for $3 \mathrm{~h} ; \mathrm{D}$, unilateral obstruction for $24 \mathrm{~h}$; E, bilateral obstruction for $24 \mathrm{~h}$; and F, immediately after release of obstruction present for $3 \mathrm{~h}$. Kidneys from these six groups were freeze clamped immediately after the P-31 NMR in vivo spectroscopy and subsequently assayed for ATP concentration.

Characterization of peak $X$. Kidneys obstructed for $3 \mathrm{~h}$ were freeze clamped and extracted and studied with P-31 NMR as described above. Kidneys were subjected to either no obstruction or unilateral ureteral ligation. $T_{1}$ values for the spectral peaks were determined. As this determination required from 4 to $6 \mathrm{~h}$, the $T_{1}$ measurement of in situ kidneys was begun $\sim 1 \mathrm{~h}$ after ureteral ligation. Urine obtained by cannulation of ureters ligated for $3 \mathrm{~h}$ was studied with P-31 NMR for spectral appearance as well as $T_{1}$ value of the major spectral peak.

\section{Statistics}

Results are expressed as the mean \pm SEM unless otherwise stated. All multiple group comparisons were performed with one-way analysis of variance. Individual group comparisons were performed with the Student's $t$ test using Bonferroni's correction for multiple comparisons (23).

\section{Results}

Time course. Shortly after ureteral ligation, all kidney spectra showed a marked accentuation of a peak present at $7096.63 \pm 0.65 \mathrm{~Hz}(n=12)$ (or $3.32 \pm 0.04 \mathrm{ppm}$ from the reference frequency of phosphocreatine). This peak achieved maximal height after 3 to $4 \mathrm{~h}$ of ureteral ligation on the unilaterally obstructed and bilaterally obstructed kidneys studied serially $(n=5)$. After 6 to $8 \mathrm{~h}$ in the unilaterally obstructed kidney model and $12 \mathrm{~h}$ in the bilaterally obstructed model, this peak began to diminish in amplitude. This peak returned to preobstruction amplitude by $24 \mathrm{~h}$ in the unilaterally obstructed kidneys but did not fully return to normal in bilaterally obstructed kidneys after $24 \mathrm{~h}$. Representative spectra of the experimental groups are shown in Figs. 1-6. Relative peak areas are tabulated in Table I. These data show clearly that the concentration

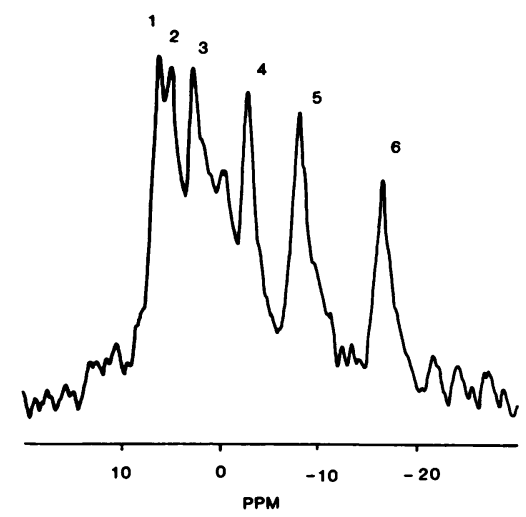

of the chemical species responsible for peak $X$ increases markedly with short periods $(3 \mathrm{~h})$ of unilateral or bilateral obstruction (groups $B$ and C). This relative concentration is significantly increased compared with normal kidneys in kidneys subjected to $3 \mathrm{~h}$ of unilateral and bilateral obstruction. Moreover, the change in the appearance of the spectra obtained are perhaps even more impressive as peak $X$ clearly becomes the dominant spectral peak after $3 \mathrm{~h}$ of obstruction (Figs. 2 and 3). This increase in peak $X$ seen with $3 \mathrm{~h}$ of unilateral obstruction is reversed immediately after release of the obstruction (group F, Fig. 6). Kidneys subjected to unilateral obstruction for $24 \mathrm{~h}$ reestablished a normal P-31 NMR spectra (group D, Fig. 4). However, kidneys subjected to $24 \mathrm{~h}$ of bilateral obstruction have persistently elevated relative concentrations of peak $X$ as well as an elevation of Pi (group E, Fig. 5). Absolute concentrations of ATP determined by enzymatic methods are indistinguishable from normal in all groups studied except the kidneys subjected to $24 \mathrm{~h}$ of bilateral obstruction, where it was significantly reduced (group E, Table II). The molar concentrations of peak $X$ determined by combining P-31 NMR and destructive methodologies (see below) confirm that the concentration of the substance responsible for peak $\mathrm{X}$ increases markedly after $3 \mathrm{~h}$ of unilateral and bilateral obstruction and returns to normal after relief of obstruction or after prolonged $(24 \mathrm{~h})$ periods of unilateral but not bilateral obstruction (Table III).

Characterization of peak $X$. P-31 NMR spectra of tissue extracts $(n=5)$ reveal no significant difference in the relative quantity of phosphodiesters between normal and obstructed kidneys. Table IV shows the relative peak areas obtained from tissue extracts of normal and obstructed kidneys. The relative area under the Pi peak in obstructed kidney extracts is signifi-

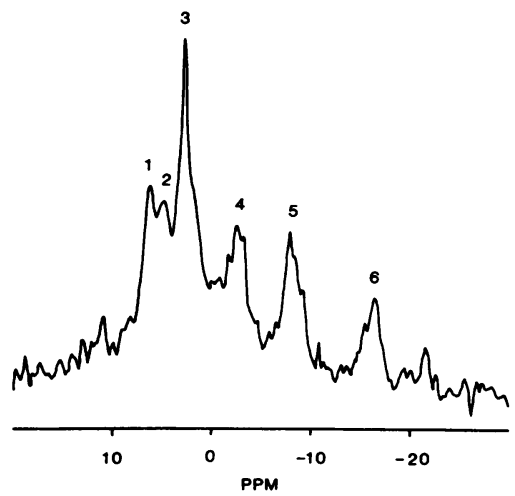

Figure 2. Group B. Spectrum of unilateral obstruction kidney (3 h). Peak assignments and acquisition parameter as in Fig. 1. 


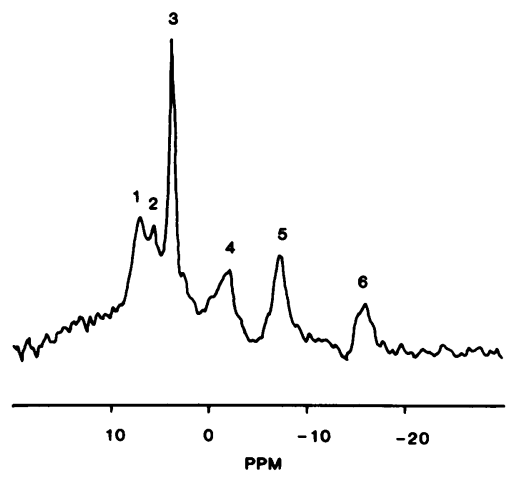

Figure 3. Group C. Spectrum of bilateral obstruction kidney ( 3 h). Peak assignments and acquisition parameter as in Fig. 1.

Figure 4. Group D. Spectrum of unilateral obstruction kidney ( 24 h). Peak assignments and acquisition parameter as in Fig. 1.

cantly greater than in normal kidneys (17.8 \pm 1.7 vs. $11.6 \pm 0.9 \%, P<0.01)$. These data were obtained with a relatively short delay $(1 \mathrm{~s})$. To rule out misinterpretation due to possible saturation, three normal and three obstructed kidney extracts were examined using $90^{\circ}$ pulses with long delays (20 s). The results obtained were similar with no significant difference in the relative concentration of phosphodiesters (12.6 \pm 1.2 vs. $16.2 \pm 2.3 \%$ ), and higher $\mathrm{Pi}$ concentrations observed in the obstructed kidneys $(16.2 \pm 2.3$ vs. $7.0 \pm 1.6 \%, P$ $<0.01)$. Using fully relaxed (20-s delays) spectra from normal $(n=5)$ and obstructed ( $3 \mathrm{~h}$ unilateral) kidneys $(n=5)$ in vivo, peak $X$ was found to increase $1.32 \pm 2.6 \mu \mathrm{mol} / \mathrm{g}$ wet weight during $3 \mathrm{~h}$ of obstruction. This value correlates relatively well with the increase in $\mathrm{Pi}$ in the fully relaxed (20-s delays) spectra from the obstructed kidney extracts $(1.46 \pm 0.49 \mu \mathrm{mol} / \mathrm{g}$ wet weight, $n=3$ ).

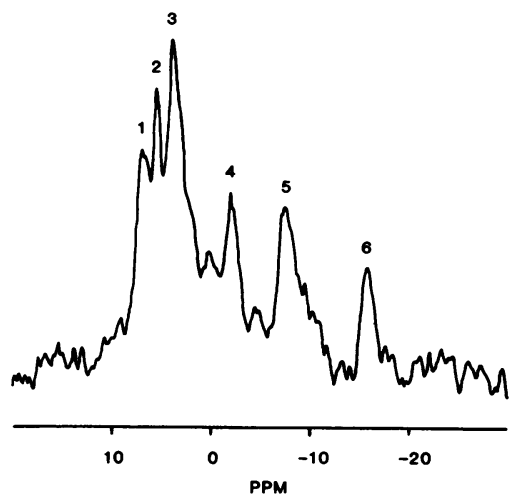

Figure 5. Group E. Spectrum of bilateral obstruction kidney ( 24 h). Peak assignments and acquisition parameter as in Fig. 1.

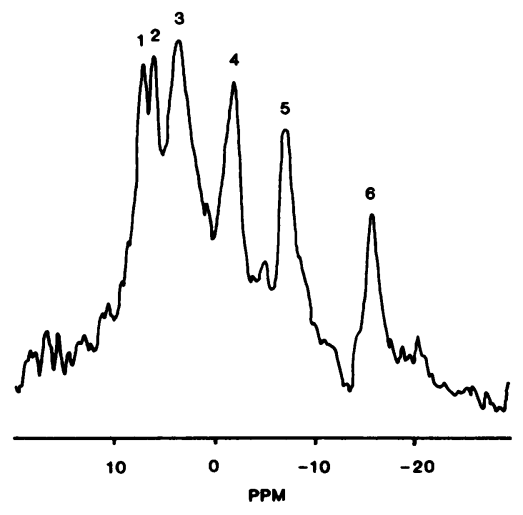

Figure 6. Group F. Spectrum of unilateral obstruction kidney $(3 \mathrm{~h})$ immediately after release of obstruction. Peak assignments and acquisition parameter as in Fig. 1.

Urine $\mathrm{pH}$ was $5.2 \pm 0.2(n=3)$ in the samples studied. Urine phosphate was $97.4 \pm 12 \mathrm{mM}$ in these samples. P-31 NMR spectra obtained from these urines after relieving the ligation showed essentially a single peak, which we attributed to urine phosphate at $7096.99 \pm 8.5 \mathrm{~Hz}$. A single peak was observed when urine $\mathrm{pH}$ was increased to 10 .

A comparison of the $T_{1}$ values for the spectral peaks of normal kidneys and kidneys subjected to unilateral ureteral ligation is shown in Table V. It is immediately clear that the longest $T_{1}$ belongs to peak $X$ in obstructed kidneys. It is also clear that the $60^{\circ}$ pulse and 2-s delay used actually underestimate the true concentration of this substance more than that of the other compounds. Of greater interest is the observation that the $T_{1}$ value of peak $X$ of obstructed kidneys is quite similar to that obtained on urine $\mathrm{Pi}(5.52 \pm 0.60$ vs. $7.14 \pm 2.69$ s, $P>0.10)$.

\section{Discussion}

P-31 NMR spectra of rat kidneys that were exposed to either unilateral or bilateral obstruction show the rapid accentuation of a peak at $\sim 7,097 \mathrm{~Hz}$ that we have designated peak $\mathrm{X}$. This peak has been attributed to various compounds by previous investigators and is almost certainly under normal conditions derived from more than one biologic compound (3-8). The

Table I. Relative Areas of P-31 Spectral Peaks

\begin{tabular}{lrlclll}
\hline Groups $(N)$ & \multicolumn{1}{c}{$S P$} & $P i$ & X & Gamma & Alpha & Beta \\
\hline A (5) & $13 \pm 1$ & $4 \pm 1$ & $5 \pm 1$ & $26 \pm 1$ & $33 \pm 2$ & $21 \pm 1$ \\
B (5) & $9 \pm 1$ & $3 \pm 1$ & $18 \pm 2^{\ddagger}$ & $21 \pm 2$ & $31 \pm 2$ & $18 \pm 2$ \\
C (3) & $7 \pm 1$ & $4 \pm 1$ & $23 \pm 2^{\ddagger}$ & $21 \pm 1^{*}$ & $29 \pm 2$ & $17 \pm 2$ \\
D (3) & $11 \pm 2$ & $4 \pm 1$ & $3 \pm 1$ & $27 \pm 2$ & $35 \pm 6$ & $21 \pm 2$ \\
E (3) & $5 \pm 1$ & $8 \pm 1^{*}$ & $12 \pm 2^{*}$ & $21 \pm 1^{*}$ & $37 \pm 1$ & $17 \pm 1$ \\
F (3) & $16 \pm 1$ & $4 \pm 1$ & $6 \pm 1$ & $23 \pm 2$ & $34 \pm 1$ & $18 \pm 2$
\end{tabular}

Data presented as percentage of total peak area with sugar phosphate (SP) + Pi + X (peak X) + gamma (gamma ATP) + alpha (alpha ATP) + beta (beta ATP) $=100 \%$. Results expressed as mean \pm SEM. Groups A through F are described in the Methods section. Spectra were baseline corrected with the baseline hump excluded from the calculation. It is assumed that the total phosphate in the sample is constant.

${ }^{*} P<0.05$ compared with normal (group A).

${ }^{\ddagger} P<0.01$ compared with normal (group A). 
Table II. Tissue Concentration of ATP after Obstruction

\begin{tabular}{ll}
\hline Group $(N)$ & ATP \\
\hline & $\mu m o l / g$ wet weight \\
A Normal (5) & $1.60 \pm 0.19$ \\
B Unilateral obstruction for $3 \mathrm{~h} \mathrm{(6)}$ & $1.47 \pm 0.26$ \\
C Bilateral obstruction for $3 \mathrm{~h} \mathrm{(3)}$ & $1.49 \pm 0.23$ \\
D Unilateral obstruction for $24 \mathrm{~h}(3)$ & $1.39 \pm 0.05$ \\
E Bilateral obstruction for $24 \mathrm{~h} \mathrm{(3)}$ & $1.12 \pm 0.08^{*}$ \\
F After release of obstruction present for $3 \mathrm{~h} \mathrm{(3)}$ & $1.46 \pm 0.21$ \\
\hline
\end{tabular}

Kidneys were freeze clamped and extracted with perchloric acid. The neutralized extract was assayed for ATP using an enzymatic method. Results expressed as mean \pm SEM. Experimental groups $A$ through $\mathrm{F}$ are described in the Methods section.

$* P<0.05$.

time course of the increase in this peak closely correlates with the well-described increases in tubular pressures and calyceal volumes noted in both the unilateral and bilateral models of ureteral obstruction in the rat (13-19, 24-26). Therefore, it appears that the changes in this peak may at least correlate with the changes in the collecting system that occurs with obstruction.

Examination of our $T_{1}$ data suggests quite strongly that the major chemical moeity responsible for the majority of peak $\mathrm{X}$ from normal kidneys is not the same as the substance resonating at 7,097 $\mathrm{Hz}$ in our obstructed kidneys. In obstructed kidneys, peak $X$ is almost certainly due to a large degree to urine phosphate. In unobstructed kidneys, urine phosphate is probably responsible for only a small portion of what other authors have called the phosphodiester peak $(27,28)$. The increase in the urine phosphate signal seen with obstruction is probably due to stasis and the increase in urine volume as described above. The position of the peak is explained by the strong $\mathrm{pH}$ dependence of the chemical shift of phosphate and is quite

Table III. Molar Concentration of Peak X in Renal Tissue In Vivo After Ureteral Ligation

\begin{tabular}{ll}
\hline Group $(N)$ & Peak X \\
\hline & $\mu m o l / g$ wet weight \\
A Normal (5) & $0.34 \pm 0.04$ \\
B Unilateral obstruction for $3 \mathrm{~h}(6)$ & $1.45 \pm 0.27^{\ddagger}$ \\
C Bilateral obstruction for $3 \mathrm{~h} \mathrm{(3)}$ & $2.00 \pm 0.34^{\ddagger}$ \\
D Unilateral obstruction for $24 \mathrm{~h} \mathrm{(3)}$ & $0.18 \pm 0.06$ \\
E Bilateral obstruction for $24 \mathrm{~h}(3)$ & $0.86 \pm 0.11^{*}$ \\
F After release of obstruction present for $3 \mathrm{~h} \mathrm{(3)}$ & $0.43 \pm 0.11$
\end{tabular}

Results expressed as mean \pm SEM. Experimental groups A through $F$ are described in the Methods section. The absolute molar concentration of the phosphate resonating at this frequency was calculated by multiplying the relative area of peak $X$ divided by the relative area of the beta ATP peak by the absolute molar concentration of ATP, as determined by enzymatic analysis performed on the extract of that kidney. The increase in peak $X$ is underestimated because of the $T_{1}$ saturation effect.

$* P<0.05$.

$\pm P<0.01$.
Table IV. Relative Peak Areas in Extracts of Normal and Obstructed Kidneys

\begin{tabular}{lcc}
\hline Peak & Normal $(n=5)$ & Obstructed $(n=5)$ \\
\hline Sugar phosphate & $28.5 \pm 1.6$ & $22.8 \pm 2.3^{*}$ \\
Pi & $11.6 \pm 0.9$ & $17.8 \pm 1.7^{\ddagger}$ \\
Phosphodiesters & $7.0 \pm 0.9$ & $7.5 \pm 1.8$ \\
Gamma ATP & $14.5 \pm 0.2$ & $13.9 \pm 0.7$ \\
Alpha ATP & $28.2 \pm 1.1$ & $27.6 \pm 1.9$ \\
Beta ATP & $10.2 \pm 0.3$ & $10.4 \pm 0.4$ \\
\hline
\end{tabular}

Results expressed as percent of total peak area. Results tabulated are mean \pm SEM.

$* P<0.05$.

${ }^{\ddagger} P<0.01$.

consistent with the resonance of $\mathrm{Pi}$ at the urine $\mathrm{pH}$ 's obtained $(1,2,29)$. A direct correlation of the magnitude of the increase in the peak with urine phosphate concentration and a change in the chemical shift of this peak with increases in urine pH can be observed (29). Chemical method for measurement of Pi concentration in aqueous solution agrees well with the levels determined by P-31 NMR (30). This assertion is supported by the $T_{1}$ data as well as by the observations that the peak disappears immediately with release of obstruction, and that $\mathrm{Pi}$ is the only phosphate containing compound present in sufficient concentration in urine to generate this signal. The decrease in this peak with prolonged obstruction in the unilateral model is almost certainly due to the reabsorption of urine and normalization of tubular pressures seen in this model $(13,16,17)$. The demonstrated capacity of distal tubular segments to reabsorb Pi supports this contention (31-35).

Other interesting observations can be drawn from our data. Kidneys subjected to prolonged bilateral obstruction have significantly lower tissue concentrations of ATP and higher relative concentrations of inorganic phosphate. This is most easily explained by decreases in renal perfusion, which result in relative ischemia in this model $(13,15,16,19,26)$. Another observation is that the $T_{1}$ of $P i$ is significantly longer in ob-

Table V. $T_{1}$ Values for Spectral Peaks Obtained in P-31 NMR Study of Normal and Obstructed Kidneys In Vivo and Urine from Obstructed Kidneys

\begin{tabular}{llll}
\hline & \multicolumn{1}{l}{$T_{1}(s)$} & & \\
\cline { 2 - 4 } Spectral peak & Normal $(n=5)$ & Obstructed $(n=5)$ & Urine $(n=3)$ \\
\hline Sugar phosphate & $2.02 \pm 0.25$ & $1.50 \pm 0.22$ & \\
Pi & $0.99 \pm 0.03$ & $1.43 \pm 0.08^{\ddagger}$ & $7.14 \pm 2.69^{*}$ \\
Peak X & $1.76 \pm 0.38$ & $5.52 \pm 0.60^{\ddagger}$ & \\
Gamma ATP & $0.94 \pm 0.05$ & $0.87 \pm 0.13$ & \\
Alpha ATP & $0.93 \pm 0.12$ & $1.15 \pm 0.34$ & \\
Beta ATP & $0.65 \pm 0.05$ & $0.95 \pm 0.18$ & \\
& & & \\
\hline
\end{tabular}

Results expressed as mean \pm SEM.

* $P<0.05$ compared with normal.

${ }^{\ddagger} P<0.01$ compared with normal. 
structed kidneys than in normal kidneys. This is consistent with the chemical exchange of $\mathrm{Pi}$ with the gamma phosphate group of ATP being a major mechanism for magnetic relaxation of $\mathrm{Pi}$. However, other physical mechanisms may be operant. Because obstructed kidneys are using very little energy for sodium transport, normally the major energy expenditure for the kidney (36), it is possible that an indirect measurement of ATP turnover rate would be different between the two groups. More accurate measurement of the differences in energy turnover rates between obstructed and nonobstructed kidneys might be accomplished with P-31 NMR by using saturation transfer techniques that were not used in this study, but will be used for future work $(22,37)$.

The observation that phosphate derived from the urine does not resonate at the frequency observed for intracellular Pi may partially explain the differences in the concentration of kidney tissue Pi determined by in vivo P-31 NMR and the destructive chemical techniques that have been well described by other workers (4, 22, 37-39). Explanations given to explain this phenomenon, which has been noted in other organs as well as kidney, include the rapid breakdown of other phosphate-containing compounds unavoidable with destructive techniques and the concept of most intracellular Pi being in a bound state and therefore not detectable by P-31 NMR, which measures more mobile chemical species. From our data, it appears likely that a portion of Pi detected by chemical extraction of kidney tissue is derived from urine phosphate, which does not resonate in situ at the same frequency as intracellular Pi because of the different chemical environments. It should be stressed that this observation only partially explains the discrepancy in Pi concentration measurement between in vivo P-31 NMR and destructive methods applied to the kidney, and cannot be extrapolated to other organs.

The P-31 NMR spectra obtained in this study were performed on animals which had their kidneys exposed. However, noninvasive NMR techniques are available that could allow for in situ renal spectroscopy without surgery (40-42). A great problem with these techniques is the certainty that the spectra obtained is indeed derived from the area of interest. It seems possible that the presence of this urine phosphate peak might complement the absence of phosphocreatine signal in assuring the spectroscopist that the spectra is indeed derived from the kidney. In particular, this may be helpful in human renal P-31 NMR spectroscopy. Moreover, if the findings presented here are confirmed in human obstruction, the rapid development of these spectral changes with obstruction suggests a potential clinical application. Human P-31 NMR spectroscopy studies of patients afflicted with ureteral obstruction are therefore awaited with great interest.

\section{Acknowledgments}

Sincere thanks are due to Martin Ashley, Chemistry Department, University of Colorado at Boulder, CO, for the kind loan of a Bruker 200 MHz NMR spectrometer, and to Robert Goldstein and Kevin Schilling for their technical assistance and to Loretta Durkin for her excellent secretarial assistance.

Dr. Shapiro was supported by National Institutes of Health (NIH) fellowship grant 1F32 AM-07731. The NMR instrument (Biospec 1.89 Tesla, Bruker Instruments and Oxford Research Systems) was funded by the NIH Biotechnology Resource Program, Division of Research Resources (grant RR-02436-01).

\section{References}

1. Moon, R. B., and J. H. Richards. 1973. Determination of intracellular pH by ${ }^{31} \mathrm{P}$ magnetic resonance. J. Biol. Chem. 248:7276-7278.

2. Hoult, D. I., S. J. W. Busby, D. G. Gadian, G. K. Radda, R. E. Richards, and P. J. Seeley. 1974. Observations of tissue metabolites using ${ }^{31}$ P NMR. Nature (Lond.). 252:285-287.

3. Radda, G. K., P. J. Bore, and B. Rajagopalan. 1984. Clinical aspects of NMR spectroscopy. Br. Med. Bull. 40:155-159.

4. Ross, B. D., D. Freeman, and L. Chan. 1986. Contributions of nuclear magnetic resonance to renal biochemistry. Kidney Int. 29:131-141.

5. Shulman, G. I., J. R. Alger, J. W. Prichard, and R. G. Shulman. 1984. Nuclear magnetic resonance spectroscopy in diagnostic and investigative medicine. J. Clin. Invest. 74:1127-1131.

6. Sehr, P., P. Bore, J. Papatheofanis, and G. K. Raddada. 1979. Nondestructive measurement of metabolites and tissue $\mathrm{pH}$ in the kidney by ${ }^{31} \mathrm{P}$ nuclear magnetic resonance. Br. J. Exp. Pathol. 60:632641.

7. Weiner, M. W., K. Burke, K. Green, D. Wemmer, N. WadeJardetsky, and O. Jardetsky. 1981. Feasibility of using ${ }^{31} \mathrm{P}$ NMR to study kidney metabolism in vivo. Kidney Int. 19:227. (Abstr.)

8. Balaban, R. S., D. G. Gadian, and G. K. Radda. 1981. Phosphorus nuclear magnetic resonance study of the rat kidney in vivo. Kidney Int. 20:575-579.

9. Chan, L., J. C. Waterton, and G. K. Radda. 1981. Study of rat kidney in vivo during hypovolemic shock by ${ }^{31}$ P NMR. Biochem. Soc. Trans. 9:239-241.

10. Chan, L., J. G. G. Ledingham, J. A. Dixon, K. R. Thulborn, J. C. Waterton, G. K. Radda, and B. D. Ross. 1982. Acute renal failure: a proposed mechanism based upon ${ }^{31} \mathrm{P}$ nuclear magnetic resonance studies in the rat. In Acute Renal Failure. N. Eliahou, editor, John Libbey \& Co. Ltd., London. 35-41.

11. Siegel, N. J., M. J. Avison, H. F. Reilly, J. R. Alger, and R. G. Shulman. 1983. Enhanced recovery of renal ATP with postischemic infusion of ATP- $\mathrm{MgCl}_{2}$ determined by ${ }^{31} \mathrm{P}$ NMR. Am. J. Physiol. 245:F530-F534.

12. Radda, G. K., J. J. H. Ackerman, P. Bore, P. Sehr, G. G. Wong, B. D. Ross, Y. Green, S. Bartlett, and M. Lowry. 1980. ${ }^{31}$ P NMR studies on kidney intracellular $\mathrm{pH}$ in acute acidosis. Int. J. Biochem. 12:600-607.

13. Wilson, D. R. 1980. Pathophysiology of obstructive nephropathy. Kidney Int. 18:281-292.

14. Harris, R. H., and J. M. Gill. 1981. Changes in glomerular filtration rate during complete ureteral obstruction in rats. Kidney Int. 19:603-608.

15. Gaudio, K. M., N. J. Siegel, J. P. Hayslett, and M. Kashgarian. 1980. Renal perfusion and intratubular pressure during ureteral occlusion in the rat. Am. J. Physiol. 238:F205-F209.

16. Moody, T. E., E. D. Vaughn, and J. Y. Gillenwater. 1977. Comparison of the renal hemodynamic response to unilateral and bilateral ureteral occlusion. Invest. Urol. 14:455-459.

17. Dal Canton, A., A. Corradi, R. Stanziale, G. Maruccio, and L. Migone. 1979. Effects of 24 hour unilateral ureteral obstruction on glomerular hemodynamics in the rat kidney. Kidney Int. 15:457-462.

18. Janike, J. R. 1970. The renal response to ureteral obstruction: a model for the study of factors which influence glomerular filtration pressure. J. Lab. Clin. Med. 76:373-382.

19. Ichikawa, I., and B. M. Brenner. 1979. Local intrarenal vasoconstrictor and vasodilator interactions in mild partial ureteral obstruction. Am. J. Physiol. 236:F131-F140.

20. Wollenberger, A., O. Ristau, and G. Schoffa. 1960. Eine einfache tecknik fur extrem schellen Abkhulung grosserer Gewebestuke. Pfluegers Arch. Eur. J. Physiol. 270:399-412.

21. Fiske, C. H., and Y. Subbarrow. 1925. The colorimetric determination of phosphorus. J. Biol. Chem. 66:375-400.

22. Freeman, D. M., L. Chan, H. Yahaya, P. Holloway, and B. D. 
Ross. 1986. Magnetic resonance spectroscopy for the determination of renal metabolic rate in vivo. Kidney Int. 30:35-42.

23. Wallenstein, S., C. L. Zucker, and J. L. Fleiss. 1980. Some statistical methods useful in circulation research. Circ. Res. 47:1-9.

24. Yarger, W. E., H. S. Aynedjian, and N. Bank. 1972. Micropuncture study of postobstructive diuresis in the rat. J. Clin. Invest. 51:625-637.

25. Tanner, G. A. 1979. Effects of kidney tubule obstruction on glomerular function in rats. Am. J. Physiol. 237:F379-F385.

26. Hsu, C. H., T. W. Kurtz, J. Rosenzweig, and J. M. Weller. 1978. Intrarenal hemodynamics and renal function in post obstructive uropathy. Invest. Urol. 15:348-351.

27. Burt, C. T., T. Glonek, and M. Bárány. 1976. Phosphorus-31 nuclear magnetic resonance detection of unexpected phophodiester in muscle. Biochemistry. 15:4850-4853.

28. Burt, C. T., T. Glonek, and M. Bárány. 1976. Analysis of phosphate metabolism, the intracellular $\mathrm{pH}$ and the state of adenosine triphosphate in intact muscle by phosphorus nuclear magnetic resonance. J. Biol. Chem. 251:2584-2591.

29. Shapiro, J. I., R. Cosby, and L. Chan. 1987. P-31 nuclear magnetic resonance spectral changes in the phosphodiester region. Society of Magnetic Resonance in Medicine. 6:1022. (Abstr.)

30. Shapiro, I. J., and L. Chan. 1987. In vivo determination of absolute molar concentrations of renal phosphorus metabolites using proton concentration as an internal standard. J. Magnetic Resonance. 75:125-128.

31. Mizgala, C. L., and G. A. Quamme. 1985. Renal handling of phosphate. Physiol. Rev. 65:431-466.

32. Amiel, C., H. Kuntziger, and G. Richet. 1970. Micropuncture study of handling of phosphate by proximal and distal nephron in normal and parathyroidectomized rat. Evidence for distal reabsorption. Pfluegers Arch. Eur. J. Physiol. 317:93-109.

33. Pastoriza-Munoz, E., D. R. Mishler, and C. Lechene. 1978.
Effect of parathyroid hormone on phosphate reabsorption in rat distal convolution. Am. J. Physiol. 235:F322-F330.

34. Peraino, R. A., and W. N. Suki. 1980. Phosphate transport by isolated rabbit cortical collecting tubule: Am. J. Physiol. 238:F358F362.

35. Shareghi, G. R., and Z. S. Agus. 1982. Phosphate transport in the light segment of the rabbit cortical collecting tubule. Am. J. Physiol. 242:F379-F384.

36. Kramer, K., and P. Deetjen. 1964. Oxygen consumption and sodium reabsorption in the mammalian kidney. In Oxygen in the Animal Organism. F. Dickens and E. Weil, editors. Pergamon Press, Oxford IUB Symposia Series. 31:337-341.

37. Freeman, D., S. Bartlett, G. Radda, and B. Ross. 1983. Energetics of sodium transport in the kidney: Saturation transfer ${ }^{31} \mathbf{P}$ NMR. Biochim. Biophys. Acta. 672:325-336.

38. Veech, R. L., J. W. R. Lawson, N. W. Cornell, and H. A. Krebs. 1979. Cytosolic phosphorylation potential. J. Biol. Chem. 254:65386547.

39. Bárány, M., K. Bárány, C. T. Burt, T. Glonek, and T. C. Myers. 1975. Structural changes in myoin during contraction and the state of ATP in the intact frog muscle. J. Supramol. Struct. 3:121-140.

40. Bogusky, R. T., M. Garwood, G. B. Matson, G. Acosta, L. D. Cowgill, and T. Schleich. 1986. Localization of phosphorus metabolites and sodium ions in the rat kidney. Magnetic Resonance Med. 3:251-261.

41. Gordon, R. E., P. E. Hanley, D. Shaw, D. G. Gadian, G. K. Radda, P. Styles, P. J. Bore, and L. Chan. 1980. Localization of metabolites in animals using ${ }^{31} \mathrm{P}$ topical magnetic resonance. Nature (Lond.). 287:736-738.

42. Bottomley, P. A. 1985. Noninvasive studies of high energy phosphate metabolism in human heart by depth resolve ${ }^{31} \mathrm{P}$ NMR spectroscopy. Science (Wash. DC). 229:769-772. 